\title{
Review of THz near-field methods
}

\author{
Hungyen Lin, Bernd M. Fischer, Samuel P. Mickan and Derek Abbott \\ Centre for Biomedical Engineering and School of Electrical and Electronic Engineering, The \\ University of Adelaide, SA 5005, Australia
}

\begin{abstract}
Terahertz (THz) imaging offers many attractive advantages over existing modalities especially in its ability to obtain spectroscopic information. In particular, $\mathrm{THz}$ spectra are extremely sensitive to small changes of the molecular structure and different isomeric and intermolecular configurations. With a comparatively longer wavelength $(0.3 \mathrm{~mm}$ at $1 \mathrm{THz})$, Rayleigh's criterion limits its use for imaging single cells and other microstructures. A method for breaking the Rayleigh's criterion and thus achieving sub-wavelength resolution is to adopt nearfield imaging techniques. This paper reviews the existing $\mathrm{THz}$ near-field methods and recent developments for identifying potential areas of research.
\end{abstract}

Keywords: Near-field, THz imaging, T-rays, scanning microscopy, ultrafast measurement

\section{INTRODUCTION}

The Terahertz $(\mathrm{THz})$ or T-ray region of the electromagnetic spectrum is loosely defined in the literature as the frequency range from 0.1 to $10 \mathrm{THz}^{*}$, situated between infrared (IR) light and microwave radiation region. Historically, $\mathrm{THz}$ radiation has been employed in astronomy for the spectral characterisation of the rotational and vibrational resonances, and thermal emission lines of simple molecules. ${ }^{1}$ Prior to the $1990 \mathrm{~s}$, the THz region was described as the $\mathrm{THz}$ gap, due to the lack of instrumentation for the generation and detection of $\mathrm{THz}$ radiation. However since then, significant progress has been made in the generation and detection instrumentation, thus making the $\mathrm{THz}$ gap evermore accessible. Terahertz imaging offers many attractive advantages over existing modalities, especially in its ability to obtain spectroscopic information and imaging of dielectric materials. For imaging microstructures, one of the major limitations of $\mathrm{THz}$ images is the low spatial resolution, as determined by Rayleigh's criterion due to the comparatively longer wavelength $(0.3 \mathrm{~mm}$ at $1 \mathrm{THz})$. Typical human cell sizes range from a few to hundreds of microns, ${ }^{2}$ depending on the type, while bacterial spores like bacillus anthracis (anthrax) are in the order of a few microns. In an effort to improve the resolution of $\mathrm{THz}$ images, various techniques have been proposed in the literature with an aim of breaking the diffraction limit. Many of the existing techniques draw inspiration from near-field scanning optical microscopy, with the near-field methods being used in conjunction with $\mathrm{THz}$ time domain spectroscopy. This paper presents an update on the recent developments along with the existing work.

\section{REVIEW}

In 2004, Yuan et al. ${ }^{3}$ presents a review of near-field imaging techniques that encompasses the principle of operation and the developments with the achieved spatial resolution. The major challenges for the each of the respective methods is also highlighted. However, pulsed $\mathrm{THz}$ near-field imaging is a rapidly growing area and some important work has been conducted since. In this contribution, we present a comprehensive review on the different $\mathrm{THz}$ near-field techniques in the literature that can broadly be classified into 3 groups: aperture, tip and electro-optic methods.

\footnotetext{
Further author information: (Send correspondence to Hungyen Lin)

Hungyen Lin: E-mail: hlin@eleceng.adelaide.edu.au, Telephone: (+61 8) 83036296

Derek Abbott: E-mail: dabbott@eleceng.adelaide.edu.au, Telephone: $(+61$ 8) 83035748

*Some texts define it as 0.3 to $30 \mathrm{THz}$
} 


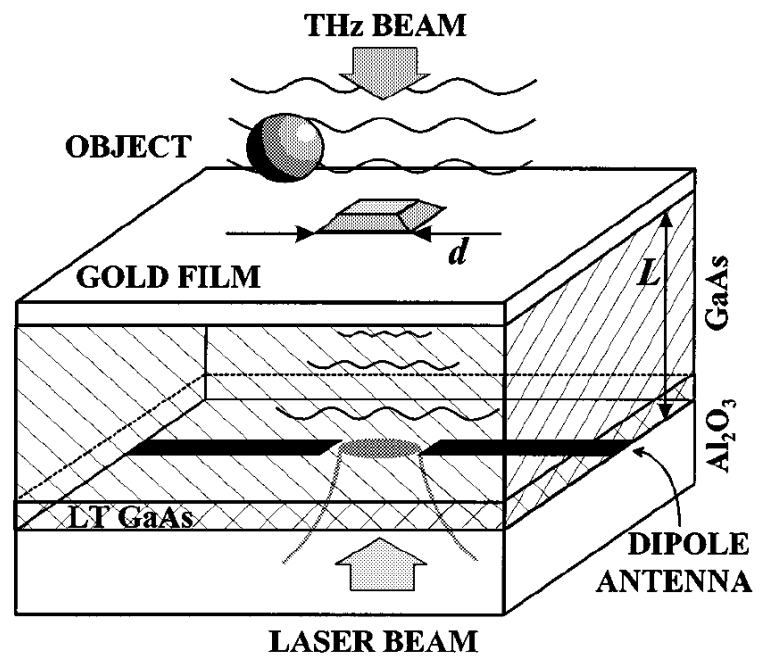

LASER BEAM

Figure 1. An example of the aperture based technique is the collection mode probe presented by Mitrofanov et al. ${ }^{8}$ The aperture is $5 \mu \mathrm{m}$ and the sample separation distance of approximately $2 \mu \mathrm{m}$. The probe achieved a resolution of $7 \mu \mathrm{m}$ with $0.5 \mathrm{THz}$ pulses in the edge test. After Mitrofanov et al. ${ }^{8}$

\subsection{Aperture based techniques}

The first demonstration of pulsed THz near-field imaging is presented by Hunsche et al., ${ }^{4}$ where an elliptical sub-wavelength aperture at the end of a tapered metal tip is used to achieve a spatial resolution approximately $50 \mu \mathrm{m}(\lambda / 4)$ in illumination mode. The 50 by $80 \mu \mathrm{m}$ aperture is placed at the focal point of the THz beam with the sample being placed in the aperture near-field. A photoconductive antenna (PCA) source is used to achieve a similar resolution by placing the sample in the near-field of the source. ${ }^{5}$ The PCA is also used in the operation of a collection or illumination mode probe on a transparent substrate with a metallic aperture to confine the generated $\mathrm{THz}$ radiation. ${ }^{6}$ A resolution of $60 \mu \mathrm{m}$ is demonstrated. With a higher refractive indexed material $\left(n_{\mathrm{GaAs}}=3.6\right)$ protruding through the aperture in a cone/pyramidal shape, the probe effectively shortens the wavelength. The probe in collector mode is demonstrated ${ }^{7}$ with a spatial resolution of approximately $40 \mu \mathrm{m}$ $(\lambda / 15)$ for a comparatively smaller spectrum and a resolution of $7 \mu \mathrm{m}$ with $0.5 \mathrm{THz}$ pulses. ${ }^{8}$

In this near-field method, the spatial resolution is no longer determined by the wavelength but the aperture size. The major limitation to this method is the low throughput resulting from the sub-wavelength aperture. Much of the radiation is reflected at the aperture plane and as shown Bethe, ${ }^{9}$ the transmitted energy through the sub-wavelength aperture of size $d$, decreases by $d^{3}$, thereby leading to a significant deterioration in the signal-to-noise ratio. For an elliptical aperture of 50 by $80 \mu \mathrm{m}$, a signal reduction factor of 130 is reported. ${ }^{4}$ The detecting element is placed in the near-field zone of the probe aperture to overcome this problem. ${ }^{10}$ This, however, is limited by the device technology. Small apertures are also affected by the metallic film thickness, which needs be sufficiently thick to reduce the incident $\mathrm{THz}$ radiation from penetration that would otherwise lead to an image background. Temporal and spectral content alteration of the transmitted $\mathrm{THz}$ pulses poses another limitation, ${ }^{10}$ while the finite thickness of the aperture exhibits waveguide effects that strongly attenuate the long wavelength components, which makes their detection difficult. Such a method is also difficult to realise in practice as it heavily relies on micro-fabrication techniques.

Another aperture based technique achieves a sub-wavelength resolution using a dynamic aperture. The technique draws inspiration from the work conducted in the mid-IR frequency range. ${ }^{11}$ An optical beam that is synchronised with the pump and probe beams, is focused at the centre of the THz beam spot, which is focused on a semiconductor wafer. The optical beam is used to generate photo-carriers on the wafer, which blocks out a sub- $\mu \mathrm{m}$ cross section of the $\mathrm{THz}$ beam thus creating a dynamic inverse aperture. ${ }^{12}$ Modulating the optical beam by means of an optical chopper, modulates the transmitted $\mathrm{THz}$ beam through the dynamic aperture, which is 


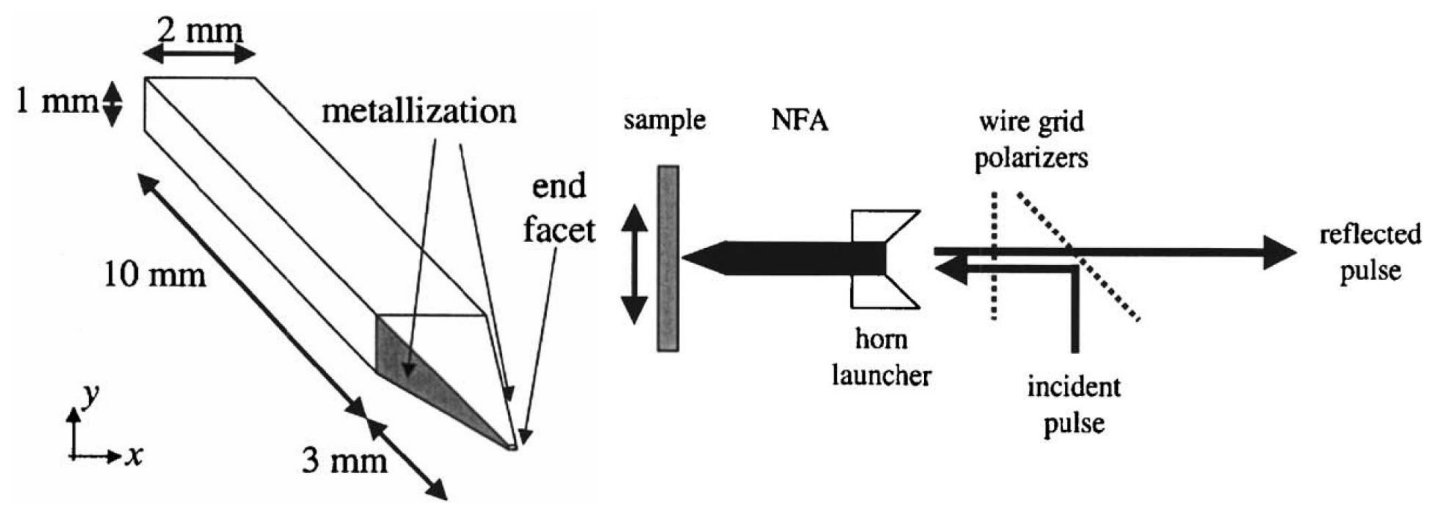

Figure 2. The low-loss dielectric waveguide with a pyramidal tip achieved a promising resolution of $20 \mu \mathrm{m}$ at $80 \mathrm{GHz}{ }^{14}$ The waveguides operates in contact with the sample. THz pulses are coupled onto the waveguide by the horn launcher and the reflected pulses are detected using electro-optic sampling. After Klein et al. ${ }^{14}$

then picked up by a lock-in amplifier. The spatial resolution of this method is determined by the focal size of the optical beam and a resolution of approximately $\lambda / 10$ has been demonstrated. ${ }^{13}$ The drawback of this method is low throughput and the need for preparation of samples with semiconductor. ${ }^{3}$

A recent development of a low-loss dielectric material waveguide with partial metalized sharpened pyramidal tip has been proposed to demonstrate a resolution of about $20 \mu \mathrm{m}$ at $80 \mathrm{GHz}(\lambda / 200) .{ }^{14}$ The waveguide operates in contact with the sample under study in both illumination and collection mode that does not involve evanescent waves. The micron-sized plane facet at the end of the pyramidal tip confines the THz wave onto a sub-wavelength spot and the reflected signal is detected by electro-optic sampling at the other end. The work has been applied for the spectroscopy of dielectric materials. ${ }^{15}$

\subsection{Tip based techniques}

The tip based method draws inspiration from the work conducted using infrared radiation ${ }^{16,17}$ and scanning near-field optical microscopy $(\mathrm{SNOM}) .{ }^{18}$ In this setup, a very sharp solid metallic tip at the end of a metallic probe is placed near the sample surface illuminated by a far-field THz beam and is mechanically oscillated. The metallic tip interacts and scatters the evanescent $\mathrm{THz}$ field in the near-field region of the sample surface for remote detection. A lock-in amplifier along with a $\mathrm{THz}$ detector is then used to measure the $\mathrm{THz}$ field at the probe oscillation frequency. The area of interaction is determined by the size of the tip which hence determines the spatial resolution.

The first THz tip based method was demonstrated by van der Valk and Planken ${ }^{19}$ with a resolution of approximately $\lambda / 110(18 \mu \mathrm{m})$. In the setup, THz pulses are focused onto a sharpened copper tip placed a few microns away from a GaP detection crystal. The polarization (linear to elliptical) of the synchronised probe beam is changed by the $\mathrm{THz}$ electric field from the tip through electro-optic effect. The amount of ellipticity in the probe polarization is proportional to the instantaneous $\mathrm{THz}$ electric field which is measured by a differential detector. Contrary to the conventional usage of $\langle 110\rangle$ oriented crystals for electro-optic detection, a $\langle 100\rangle$ oriented crystal is used instead to account for the perpendicular $\mathrm{THz}$ electric field from the tip. The probe polarization is hence modulated by the electric field that is perpendicular to the crystal surface instead of the parallel incident $\mathrm{THz}$ polarization. The resolution is determined by the spot size of the probe laser. A similar setup is implemented with a sphere instead of a tip ${ }^{20}$ for near-field measurements.

Another THz tip based method that is similar to a SNOM setup is presented by Wang et al. ${ }^{21}$ The setup uses a vibrating copper tip (head diameter $5 \mu \mathrm{m}$ ) placed in the near-field of the sample surface that is illuminated by a $\mathrm{THz}$ beam from PCA source. The scattered $\mathrm{THz}$ radiation is detected near the sample surface using a photoconductive dipole antenna. ${ }^{3,21}$ A similar adaptation of a SNOM setup is also presented by Chen et al. ${ }^{22}$ where a resolution of $150 \mathrm{~nm}$ for $2 \mathrm{THz}$ pulses $\lambda / 1000$ is achieved. A THz beam is focused to a $500 \mu \mathrm{m}$ spot size 


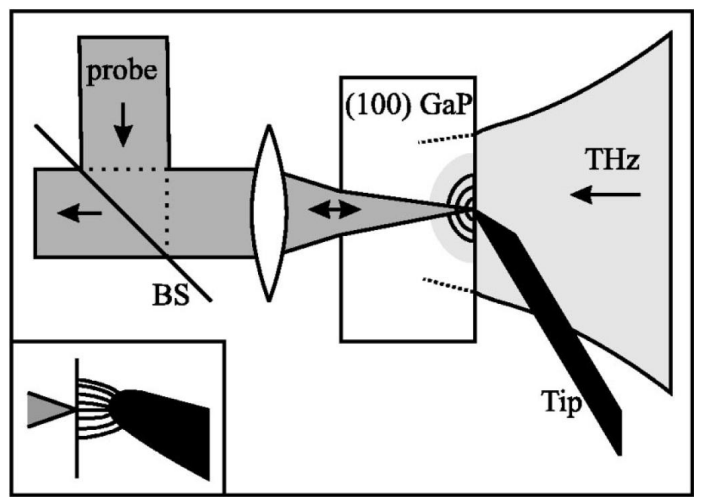

Figure 3. A tip based method with electro-optic detection achieved a resolution of $18 \mu \mathrm{m} .{ }^{19}$ This technique places the sample in close proximity to the detector (electro-optic or semi conducting medium). A synchronised probe pulse is focused into the crystal and the polarisation is changed by the electro-optic effect at the tip, and reflects back for detection. After van der Valk and Planken. ${ }^{19}$

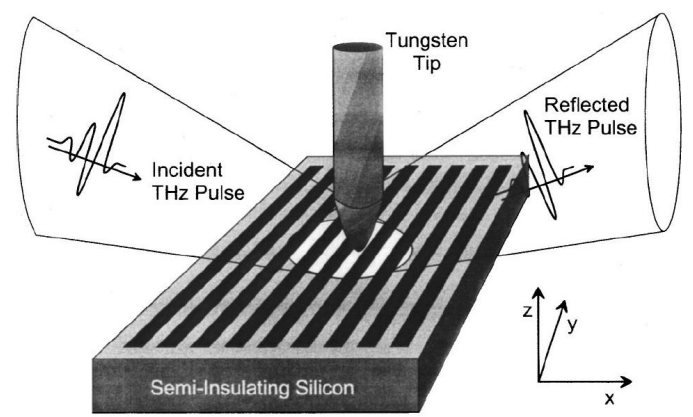

Figure 4. Another tip based method ${ }^{22}$ achieved a resolution of $10 \mu \mathrm{m}$. The tip has a radius of $100 \mathrm{~nm}$ placed $200 \mathrm{~nm}$ above the sample surface and the transmitted $\mathrm{THz}$ radiation is detected by electro-optic sampling or bolometer. After Chen et al. ${ }^{22}$

on a semi-insulating silicon substrate surface lined with $10 \mu \mathrm{m}$ wide metallic stripes. A sharpened tungsten tip is placed about $200 \mathrm{~nm}$ above the sample surface with a radius of $100 \mathrm{~nm}$. The transmitted radiation is measured by electro-optic sampling. A similar setup that implements atomic force microscopy in conjunction with $\mathrm{THz}$ TDS reports a resolution of $10 \mathrm{~nm} .{ }^{23}$ Recent reports on probing the edge between GaAs and Au achieved a lateral resolution of $80 \mathrm{~nm} .{ }^{24}$ Instead of illuminating the sample with the generated THz radiation, Yuan el al. ${ }^{25}$ achieved a sub $100 \mathrm{~nm}$ spatial resolution by modulating a metal tip in close contact $(<100 \mathrm{~nm})$ or in contact with a semiconductor surface in a weakly focused pump light spot. The purpose of the tip is to modulate the emitted $\mathrm{THz}$ pulses from small confined areas and subsequently detected by EO sampling to a lock-in amplifier. The method, however, works for a semiconductor sample for the generation of THz radiation. Bearing a similar setup, but without the tip and also for semiconductor samples, the laser THz emission microscope (LTEM) ${ }^{26}$ achieves a resolution below $3 \mu \mathrm{m}^{27}$ for inspecting electrical faults in integrated circuits. Instead of the tip, laser pulse is focused to a spot size of about $2.5 \mu \mathrm{m}^{27}$ with the backscattered T-rays collimated and focused for detection by a bow-tie antenna.

The tip based near-field approach shows a great promise in high-resolution surface topographic and $\mathrm{THz}$ spectral imaging. However, it is complicated with the introduction of a near-field tip, and suffers from low output power. Furthermore, some of the presented techniques are only valid for semiconductor samples. Chen et al. ${ }^{22}$ demonstrate the need to further refine the existing spherical scatter model for the imaging tip ${ }^{16}$ and the 
$250 \mu \mathrm{m} \mathrm{ZnTe}$

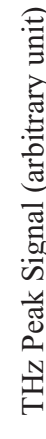

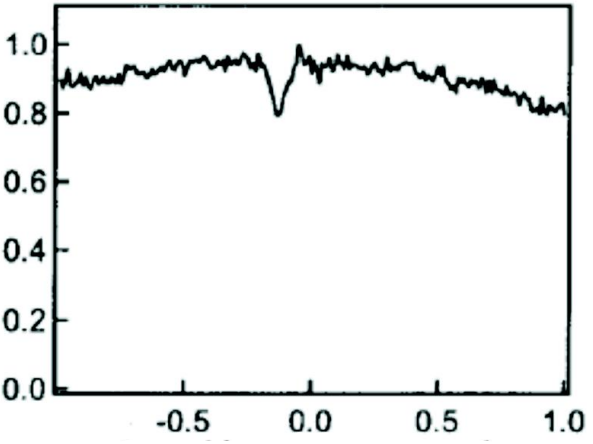

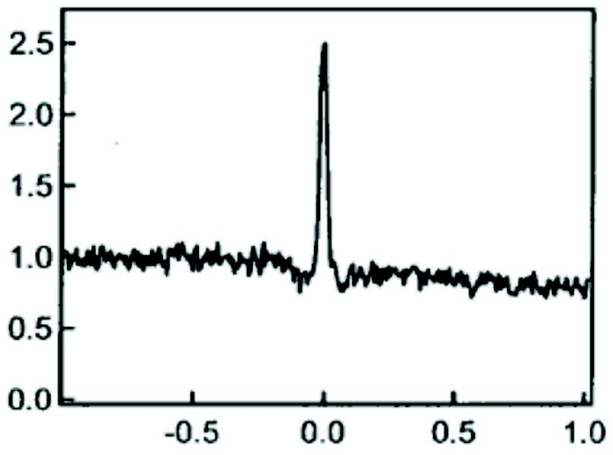

Objective Lens Position (mm)

Figure 5. Experimental result from Yuan et al. ${ }^{31}$ A thin crystal lead to an unexpected enhancement in the emitted THz power compared to the thick crystal at the focal point of the objective lens. Outside the focal point however, the emitted $\mathrm{THz}$ signal of both crystals appear to be constant with the thick being greater than the thin crystal. After Yuan et al. ${ }^{31}$
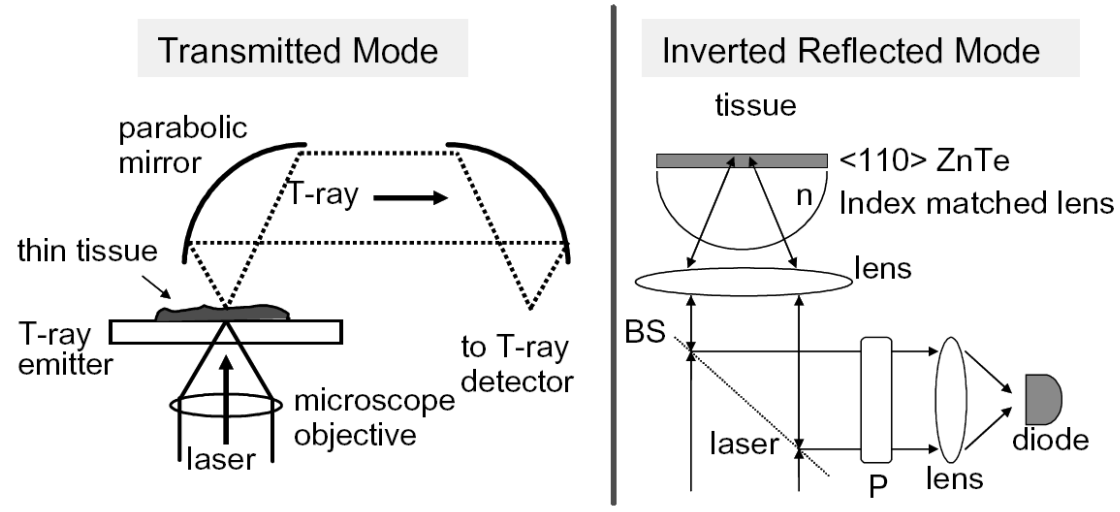

Figure 6. Schematics of the electro-optic THz microscope in transmission and reflection mode respectively. After Yuan et al. ${ }^{3}$

inclusion of antenna properties for the probe that are discussed in. ${ }^{28,29}$ An investigation on $\mathrm{THz}$ wave guiding on metal wires can be found in Wang and Mittleman. ${ }^{30}$

\subsection{Electro-optic techniques}

An alternative apertureless near-field imaging technique achieves sub-wavelength resolution by exploiting the fact that the $\mathrm{THz}$ emission point is smaller than the $\mathrm{THz}$ wavelength. This can be realised by placing the sample close to the emitter, so that the radiation would not have time to diffract before interacting with the sample. ${ }^{32}$ Wynne and Jaroszynski ${ }^{33}$ tightly focused an optical beam to a small spot in an optical rectification crystal (ZnTe) for the generation of a sub-wavelength diameter $\mathrm{THz}$ beam in the near-field region. The achievable resolution would be $\lambda / 4.3$, provided the sample is placed on the crystal. Yuan et al. ${ }^{31}$ focused near-IR ultra-fast laser to a small spot with an aim of generating $\mathrm{THz}$ signals of a spatial resolution close to the focus spot size. It is demonstrated that the generated $\mathrm{THz}$ signal at the focus point by very thin crystal $(16 \mu \mathrm{m})$ differs to the thicker counterpart $(250 \mu \mathrm{m}){ }^{31}$

Thin crystals generate a strong peak $\mathrm{THz}$ signal at the focal point as opposed to the thick crystal, which reduces the $\mathrm{THz}$ signal. The strong peak for the thin crystal is unexpected and is shown to be linearly dependent 


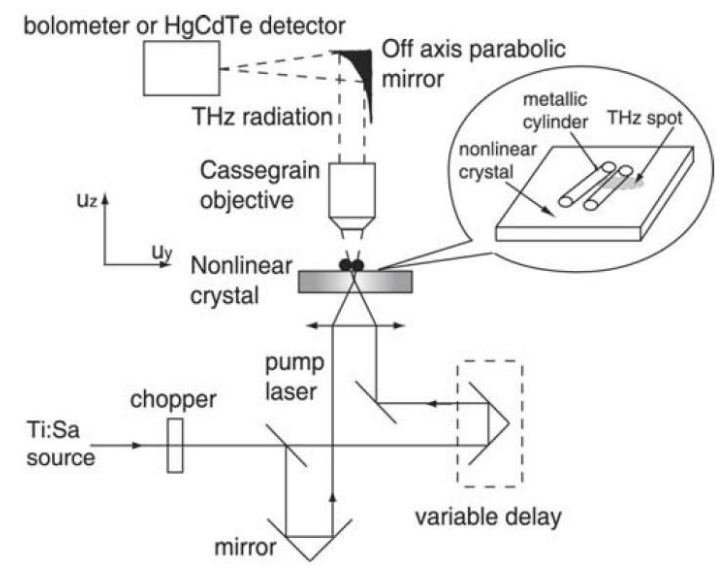

Figure 7. The electro-optic experimental setup that achieved $75 \mu \mathrm{m}$ resolution with a $500 \mu \mathrm{m}$ thick ZnTe. ${ }^{34}$ After Lecaque et al. ${ }^{34}$

on the pump power, provided it is less than $15 \mathrm{~mW}$. The intensity of the pump beam however, is limited by the two photon damage threshold of the EO crystal. A thickness in the order of tens of $\mu \mathrm{m}$ is essential to achieve a high resolution. This thickness in turn imposes physical constraints on experimentation and possible future application as thin crystals are thermally and mechanically fragile. A spatial resolution of $20 \mu \mathrm{m}$ is reported. ${ }^{3}$

Similar work is presented recently with a $500 \mu \mathrm{m}$ thick crystal for demonstrating a resolution of approximately $75 \mu \mathrm{m}$ on imaging a sharpened tungsten wire. ${ }^{34}$ The resolution is reported to be equal to the pump beam focused size. The advantage of the electro-optic methods is throughput and waveform independency of the sample topography. ${ }^{3,34}$

\section{FUTURE DIRECTIONS}

Existing work can be classified into 3 classes of techniques, namely aperture, tip and electro-optic based methods. Even though the tip method achieves the highest spatial resolution, the radiation throughput suffers from a reduction of $r^{6} / \lambda^{4}$ with decreasing tip size. ${ }^{9}$ This hence places a limitation on the spatial resolution. Contrary to the aperture and tip method, the EO approach is the more efficient alternative with the THz power decreasing proportionally to the second power of the source dimension. ${ }^{35}$ To date, limited research has been carried out in the area of near-field imaging. The unexpected signal enhancement from thin crystals still needs to be explained and understood. Another promising technique includes the dielectric antenna, ${ }^{14}$ which unlike the tip method, demonstrates a greater power efficiency.

\section{CONCLUSION}

This paper has surveyed and reviewed the existing techniques in the literature for limiting the $\mathrm{THz}$ exposure area to achieve a higher spatial resolution. The electro-optic technique promises to be a potential area of investigation for the research group with the use of thin generation crystals. A recent study on the effect of phase mismatching on crystal thickness and pulse wavelength ${ }^{36}$ indicates that phase matching might explain the unexpected signal enhancement in thin crystals. Thin crystals, however, pose many experimental difficulties that limit their practical significance. Work is therefore currently under way to better understand phase matching in optical rectification with different nonlinear crystals. Future studies aims to improve the practicality of the electro-optic technique.

\section{ACKNOWLEDGMENTS}

The authors gratefully thank for the support of the Australian Research Council and the Sir Ross and Sir Keith Smith Fund. 


\section{REFERENCES}

1. P. Siegel, "Terahertz technology in biology and medicine," IEEE Transactions on Microwave Theory and Techniques 52(10), pp. 2438-47, 2004.

2. A. J. Vander, J. H. Sherman, and D. S. Luciano, Human Physiology: The Mechanisms of Body Function, McGraw-Hill Publishing Company, 1990.

3. T. Yuan, J. Xu, and X.-C. Zhang, "Development of terahertz wave microscopes," Infrared Physics and Technology 45, pp. 417-25, 2004.

4. S. Hunsche, M. Koch, I. Brener, and M. Nuss, "THz near-field imaging," Optics Communications 150, pp. 22-6, 1998.

5. I. Brener, S. Hunsche, Y. Cai, M. Nuss, J. Wynn, J. Lopata, and L. Pfeiffer, "Time resolved near field imaging with sub-wavelength far-infrared dipole sources," in Ultrafast Phenomena XI, Springer Series in Chemical Physics 63, pp. 171-173, Springer-Verlag, (Berlin), 1998.

6. O. Mitrofanov, I. Brener, M. Wanke, R. Ruel, J. Wynn, A. Bruce, and J. Federici, "Near-field microscope probe for far infrared time domain measurements," Applied Physics Letters 77(4), pp. 591-593, 2000.

7. O. Mitrofanov, I. Brener, R. Harel, J. Wynn, L. Pfeiffer, K. West, and J. Federici, "Terahertz near-field microscopy based on a collection mode detector," Applied Physics Letters 77(22), pp. 3496-8, 2000.

8. O. Mitrofanov, M. Lee, J. Hsu, I. Brener, R. Harel, J. Federici, J. Wynn, L. Pfeiffer, and K. West, "Collectionmode near-field imaging with 0.5-THz pulses," IEEE Journal of Selected Topics in Quantum Electronics 7, pp. $600-7,2001$.

9. H. A. Bethe, "Theory of diffraction by small holes," Phys. Rev. 66, pp. 163-182, 1944.

10. O. Mitrofanov, M. Lee, J. Hsu, L. Pfeiffer, K. West, J. Wynn, and J. Federici, "Terahertz pulse propagation through small apertures," Applied Physics Letters 79(7), pp. 907-9, 2001.

11. D. Palanker, G. Knippels, T. Smith, and H. Schwettman, "IR microscopy with a transient photo-induced near-field probe (tipless near-field microscopy)," Optics Communications 148, pp. 215-20, 1998.

12. Q. Chen, Z. Jiang, G. Xu, and X.-C. Zhang, "Near-field terahertz imaging with a dynamic aperture," Optics Letters 25, pp. 1122-4, 2000.

13. Q. Chen and X.-C. Zhang, "Semiconductor dynamic aperture for near-field terahertz wave imaging," IEEE Journal of Selected Topics in Quantum Electronics 7, pp. 608-14, 2001.

14. N. Klein, P. Lahl, U. Poppe, F. Kadlec, and P. Kuzel, "A metal-dielectric antenna for terahertz near-field imaging," Journal of Applied Physics 98(1), pp. 14910-14910, 2005.

15. M. Berta, S. Danylyuk, F. Kadlec, P. Kuzel, and N. Klein, "THz near-field spectroscopy based on metal dielectric antennae," in Conference Digest of the 2006 Joint 31st International Conference on Infrared and Millimeter Waves and 14th International Conference on Terahertz Electronics, p. 373, (Shanghai, China), 2006.

16. B. Knoll and F. Keilmann, "Near-field probing of vibrational absorption for chemical microscopy," $N a-$ ture 399(6732), pp. 134-7, 1999.

17. R. Hillenbrand, T. Taubner, and F. Keilmann, "Phonon-enhanced light-matter interaction at the nanometre scale," Nature 417, pp. 159-62, 2002.

18. F. Zenhausern, Y. Martin, and H. Wickramasinghe, "Scanning interferometric apertureless microscopy: optical imaging at 10 angstrom resolution," Science 269(5227), pp. 1083-5, 1995.

19. N. van der Valk and P. Planken, "Electro-optic detection of subwavelength terahertz spot sizes in the near field of a metal tip," Applied Physics Letters 81(9), pp. 1558-60, 2002.

20. A. Adam, J. Brok, A. van de Nes, and P. Planken, "Terahertz near-field measurements of field enhancement near metal objects," in Conference Digest of the 2006 Joint 31st International Conference on Infrared and Millimeter Waves and 14th International Conference on Terahertz Electronics, p. 15, (China), 2006.

21. K. Wang, A. Barkan, and D. Mittleman, "Sub-wavelength resolution using apertureless terahertz near-field microscopy," in Conference on Lasers and Electro-Optics (CLEO), p. 2, (Baltimore, MD, USA), 2003.

22. H.-T. Chen, R. Kersting, and G. C. Cho, "Terahertz imaging with nanometer resolution," Applied Physics Letters 83, pp. 3009-3011, 2003. 
23. R. Hillenbrand and F. Keilmann, "Material-specific mapping of metal semiconductor dielectric nanosystems at $10 \mathrm{~nm}$ resolution by backscattering near-field optical microscopy," Applied Physics Letters 80, pp. 25-7, 2002.

24. H. Park, J. Kim, M. Kim, and H. Hanl, "Terahertz near-field microscope," in Conference Digest of the 2006 Joint 31st International Conference on Infrared and Millimeter Waves and 14th International Conference on Terahertz Electronics, p. 13, (Shanghai, China), 2006.

25. T. Yuan, H. Park, J. Xu, H. Han, and X.-C. Zhang, "Field induced THz wave emission with nanometer resolution," Proceedings of SPIE - The International Society for Optical Engineering 5649(Part 1), pp. 1-8, (Sydney, Australia), 2005.

26. T. Kiwa, M. Tonouchi, M. Yamashita, and K. Kawase, "Laser terahertz-emission microscope for inspecting electrical faults in integrated circuits," Optics Letters 28, pp. 2058-60, 2003.

27. M. Yamashita, T. Kiwa, M. Tonouchi, K. Nikawa, C. Otani, and K. Kawase, "Laser terahertz emission microscope for inspecting electrical failures in integrated circuits," International Meeting for Future of Electron Devices, Kansai (IEEE Cat. No. 04EX829), pp. 29-30, (Kyoto, Japan), 2004.

28. K. Wang, A. Barkan, and D. Mittleman, "Propagation effects in apertureless near-field optical antennas," Applied Physics Letters 84(2), pp. 305-7, 2004.

29. K. Wang, D. M. Mittleman, N. C. van der Valk, and P. C. Planken, "Antenna effects in terahertz apertureless near-field optical microscopy," Applied Physics Letters 85(14), pp. 2715-2717, 2004.

30. K. Wang and D. M. Mittleman, "Metal wires for terahertz wave guiding," Nature 432(7015), pp. 376-379, 2004.

31. T. Yuan, S. Mickan, J. Xu, D. Abbott, and X.-C. Zhang, "Towards an apertureless electro-optic T-ray microscope," Pacific Rim Conference on Lasers and Electro-Optics, CLEO - Technical Digest , pp. 637-638, (Long Beach, CA, United States), 2002.

32. D. Mittleman, M. Gupta, R. Neelamani, R. Baraniuk, J. Rudd, and M. Koch, "Recent advances in terahertz imaging," Applied Physics B (Lasers and Optics) B68(6), pp. 1085-94, 1999.

33. K. Wynne and D. Jaroszynski, "Superluminal terahertz pulses," Optics Letters 24(1), pp. 25-7, 1999.

34. R. Lecaque, S. Gresillon, N. Barbey, R. Peretti, J.-C. Rivoal, and C. Boccara, "THz near-field optical imaging by a local source," Optics Communications 262(1), pp. 125-128, 2006.

35. J. Xu and X.-C. Zhang, "Optical rectification in an area with a diameter comparable to or smaller than the center wavelength of terahertz radiation," Optics Letters 27, pp. 1067-9, 2002.

36. N. van der Valk, P. Planken, A. Buijserd, and H. Bakker, "Influence of pump wavelength and crystal length on the phase matching of optical rectification," Journal of the Optical Society of America B (Optical Physics) 22, pp. 1714-18, 2005. 